\title{
Cosmic absorption of ultra high energy particles
}

\author{
R. Ruffini ${ }^{a, b, c}$ G.V. Vereshchagin, ${ }^{a, b, 1}$ S.-S. Xue $\mathbf{e}^{a, b}$ \\ ${ }^{a}$ ICRANet Piazzale della Repubblica, 10 -65122, Pescara \\ ${ }^{b}$ ICRA and Department of Physics, University of Rome "Sapienza", P.le A. Moro 5, 00185 \\ Rome, Italy \\ ${ }^{c}$ ICRANet-Rio, Centro Brasileiro de Pesquisas Fisicas, Rua Dr. Xavier Sigaud 150, Rio de \\ Janeiro, 22290180, Brazil \\ E-mail: ruffini@icra.it, veresh@icra.it, xue@icra.it
}

\begin{abstract}
This paper summarizes the limits on propagation of ultra high energy particles in the Universe, set up by their interactions with cosmic background of photons and neutrinos. By taking into account cosmic evolution of these backgrounds and considering appropriate interactions we derive the mean free path for ultra high energy photons, protons and neutrinos. For photons the relevant processes are the Breit-Wheeler process as well as the double pair production process. For protons the relevant reactions are the photopion production and the Bethe-Heitler process. We discuss the interplay between the energy loss length and mean free path for the Bethe-Heitler process. Neutrino opacity is determined by its scattering off the cosmic background neutrino. We compute for the first time the high energy neutrino horizon as a function of its energy.
\end{abstract}

\footnotetext{
${ }^{1}$ Corresponding author.
} 


\section{Contents}

1 Introduction $\quad 1$

2 Processes 2

2.1 Processes involving photons 2

2.2 Processes involving protons 3

2.3 Processes involving neutrinos 4

3 The optical depth and the mean free path 5

4 The mean energy loss distance $\quad 6$

5 Results $\quad 7$

$\begin{array}{lll}5.1 & \text { Photons } & 7\end{array}$

$\begin{array}{llr}5.2 \text { Protons } & 10\end{array}$

$\begin{array}{lll}5.3 & \text { Neutrinos } & 12\end{array}$

6 Conclusions 13

\section{Introduction}

Observation of ultra high energy (UHE) particles, such as photons, ions and neutrinos, provides the crucial information on astrophysical systems as well as mechanisms of charged particle acceleration in these systems. Such information cannot be obtained from the study of low energy emission, although it is much easier to detect.

Propagation of UHE particles on cosmological distances involves interaction with other particles, as well as with electromagnetic fields, in the case of charged particles [1]. One of the most important reservoir of photons is the cosmic microwave background (CMB). Interaction with CMB imposes strong limits on propagation of UHE photons, protons, and nuclei. Extragalactic background light (EBL), being the accumulated radiation in the Universe due to stars and active galactic nuclei, represents additional background of photons [2], which limits propagation of high energy photons. Yet another important background is cosmic neutrino background $(\mathrm{C} \nu \mathrm{B})$, which places a tight limit on the propagation of UHE neutrinos.

In this work, we review stringent limits on propagation of UHE particles, namely photons, protons and neutrinos, in the Universe due to their interactions with cosmic background of photons and neutrinos. We pay the particular attention to accounting for the cosmic evolution of $\mathrm{CMB}$ and $\mathrm{C} \nu \mathrm{B}$ fields, being important at high redshifts. We discuss relation with previous results, as well as implications of new results, obtained in this work.

First in Sec. 2 we discuss most important processes, responsible for interaction of UHE particles with cosmic backgrounds, as well as the corresponding cross-sections. Most of these processes are discussed in detail in [3]. Then in Sec. 3 we introduce the method used to compute the mean free path of UHE particles, which takes into account cosmological redshift of particle energy, as well as temperature evolution of the CMB and C $\nu$ B. In Sec. 4 the definition of the mean energy loss distance is given. We present and discuss results in Sec. 5 . Conclusions follow. 


\section{Processes}

\subsection{Processes involving photons}

UHE photons are likely produced in sources of UHE cosmic rays. The most important process, responsible for intergalactic absorption of high-energy $\gamma$-rays is the Breit-Wheeler process [4] for the photon-photon pair production

$$
\gamma_{1}+\gamma_{2} \longrightarrow e^{+}+e^{-}
$$

It was first discussed by Nikishov [5] back in 1961 and then, after the discovery of CMB, by Gould and Schreder [6].

Breit and Wheeler [4] studied collision process (2.1) of two photons with energies $E$ and $\mathcal{E}$ in the laboratory frame, producing electron and positron pair. They found the total cross-section

$$
\sigma_{\gamma \gamma}=\frac{\pi}{2}\left(\frac{\alpha \hbar}{m_{e} c}\right)^{2}\left(1-\beta^{2}\right)\left[2 \beta\left(\beta^{2}-2\right)+\left(3-\beta^{4}\right) \ln \left(\frac{1+\beta}{1-\beta}\right)\right],
$$

where

$$
\beta=\sqrt{1-\frac{1}{x}}, \quad x=\frac{E \mathcal{E}}{\left(m_{e} c^{2}\right)^{2}},
$$

$\hbar$ is Planck's constant, $m_{e}$ is electron mass, $c$ is the speed of light and $\alpha$ is the fine structure constant. The necessary kinematic condition in order for the process (2.1) to take place is that the energy of two colliding photons is larger than the energetic threshold $2 m_{e} c^{2}$, i.e., $x \geq 1$. Due to this kinematic condition the function (2.2) has a low energy cut-off at $x=1$. The cross-section has a maximum at $x \simeq 2$, with $\sigma_{\gamma \gamma}^{\max } \simeq \sigma_{T} / 4$, where $\sigma_{T}$ is Thomson cross-section. At higher energies it decreases as $1 / x$.

A simple estimate of the mean free path for the Breit-Wheeler absorption of high energy photon can be given as follows. Considering the actual CMB photon density $n_{C M B} \simeq 411$ $\mathrm{cm}^{-3}$ and taking $\sigma_{T} / 4$ for the cross-section of the interaction, the mean free path is $\lambda_{B W}=$ $\left(\sigma_{T} n_{C M B} / 4\right)^{-1} \simeq 4.8 \mathrm{kpc}$. One can refer to this distance as to a horizon, namely the maximal distance to the source for which the particle with the given energy can still be detected on Earth. However, owing to the energy dependence of the cross-section, and cosmic evolution of the CMB photon field the actual mean free path strongly depends on energy. The characteristic energy of UHE photons interacting with CMB, having temperature today $T_{0} \approx 2.725 \mathrm{~K}$, is given by $E_{B W}=\left(m_{e} c^{2}\right) / k T_{0} \simeq 1.11 \mathrm{PeV}$. At lower energies, in the $\mathrm{TeV}$ range, photons interact by the Breit-Wheeler process with the EBL [6-8]. Hence the observation of $\mathrm{TeV}$ radiation from distant $(d>100 \mathrm{Mpc})$ extragalactic objects provides important constraints on the EBL [9-11].

At much higher energies the double pair production process

$$
\gamma_{1}+\gamma_{2} \rightarrow e^{+}+e^{-}+e^{+}+e^{-}
$$

dominates, see $[12,13]$. In this high-energy limit it has nearly a constant cross-section, see e.g. [3]

$$
\sigma_{d p p}=\frac{\alpha^{2}}{36 \pi}\left(\frac{\alpha \hbar}{m_{e} c}\right)^{2}[175 \zeta(3)-38] \sim 6.5 \mu b .
$$

Clearly, this process has a threshold with the sum of energies of photons which must exceed $4 m_{e} c^{2}$. It imposes a limit for UHE photons propagation $\lambda_{d p p}=\left(\sigma_{d p p} n_{C M B}\right)^{-1} \simeq 121 \mathrm{Mpc}$. 


\subsection{Processes involving protons}

Charged UHE particles, such as protons and nuclei, are assumed to originate from extragalactic sources, which work as "cosmic accelerators" [1]. Such particles interact with the CMB photons as well. In fact, the famous Greisen-Zatsepin-Kuzmin (GZK) limit [14, 15] was established by considering that this UHE particle interacts with the CMB photons via the pion photoproducton process

$$
p+\gamma \longrightarrow\left(\begin{array}{l}
p \\
n
\end{array}\right)+\pi
$$

and lose its initial energy. Due to the fact that at this process the proton loses more than half of its energy [16], such interaction imposes a strong cut-off on energies of UHE cosmic rays. The cut-off energy is easy to estimate. Recall that the characteristic energy in the Breit-Wheeler process (2.1) is $E_{B W}=\left(m_{e} c^{2}\right)^{2} / k T_{0}$. When the photopion process (2.6) is concerned, the electron mass is exchanged with the pion mass, and an additional factor 4 comes from the reference frame transformation, giving $E_{p \gamma}=4\left(m_{\pi} c^{2}\right)^{2} / k T_{0} \simeq 3 \times 10^{5} E_{B W}=$ $3.33 \times 10^{20} \mathrm{eV}$. More careful evaluation of the energy by comparing energy losses due to photopion (2.6) and photoproduction of pair (2.8) processes (see below) gives the value $E_{p \gamma}=5 \times 10^{19} \mathrm{eV}$ [17]. The cross-section of the photopion process in high energy limit is constant with the value $[16]$

$$
\sigma_{p \gamma} \simeq 120 \mu b .
$$

The mean free path due to this process for energies $E>E_{p \gamma}$ is $\lambda_{p \gamma}=\left(\sigma_{p \gamma} n_{C M B}\right)^{-1} \simeq 6$ Mpc.

Another process relevant for interaction of UHE particles with CMB is the photoproduction of electron-positron pair on a nucleus, or Bethe-Heitler process [18]. In the case of proton, which is the only one considered in this work, this process is

$$
p+\gamma \longrightarrow p+e^{+}+e^{-} .
$$

It has the characteristic energy $E_{B H}=m_{e} m_{p} c^{4} /\left(2 k T_{0}\right) \simeq 1.0 \times 10^{18} \mathrm{eV}$. This process has a threshold with photon energy in the proton rest frame $\mathcal{E}^{\prime}>2 m_{e} c^{2}$. We use for its crosssection in the proton rest frame the expressions given in [19], namely near the threshold with $2 \leq \epsilon^{\prime} \leq 4$

$$
\sigma_{B H}^{t h r}\left(\epsilon^{\prime}\right) \simeq \frac{2 \pi}{3} \alpha\left(\frac{\alpha \hbar}{m_{e} c}\right)^{2}\left(\frac{\epsilon^{\prime}-2}{\epsilon^{\prime}}\right)^{3}\left(1+\frac{1}{2} \eta+\frac{23}{40} \eta^{2}+\frac{37}{120} \eta^{3}+\frac{61}{192} \eta^{4}\right)
$$

where $\epsilon^{\prime}=\mathcal{E}^{\prime} /\left(m_{e} c^{2}\right)$ is photon energy in the proton rest frame and $\eta=\left(\epsilon^{\prime}-2\right) /\left(\epsilon^{\prime}+2\right)$. At higher energies $\epsilon^{\prime}>4$ the cross-section is

$$
\begin{gathered}
\sigma_{B H}^{h e}\left(\epsilon^{\prime}\right) \simeq \alpha\left(\frac{\alpha \hbar}{m_{e} c}\right)^{2}\left\{\frac{28}{9} \delta-\frac{218}{27}+\left(\frac{2}{\epsilon^{\prime}}\right)^{2}\left[6 \delta-\frac{7}{2}+\frac{2}{3} \delta^{3}-\delta^{2}-\frac{\pi^{2}}{3} \delta+2 \zeta(3)+\frac{\pi^{2}}{6}\right]\right. \\
\left.-\left(\frac{2}{\epsilon^{\prime}}\right)^{4}\left[\frac{3}{16} \delta+\frac{1}{8}\right]-\left(\frac{2}{\epsilon^{\prime}}\right)^{6}\left[\frac{29}{9 \times 256} \delta-\frac{77}{27 \times 512}\right]\right\},
\end{gathered}
$$

where $\delta=\log \left(2 \epsilon^{\prime}\right)$. Expression (2.10) is logarithmically increasing at high energies, so we can take a characteristic value obtained by Bethe and Heitler $\sigma_{B H} \simeq(28 / 9) \alpha\left[(\alpha \hbar) /\left(m_{e} c\right)\right]^{2}$ in 
order to estimate the mean free path of UHE protons, which gives $\lambda_{B H}=\left(\sigma_{B H} n_{C M B}\right)^{-1} \simeq$ $437 \mathrm{kpc}$.

It is important to note that unlike the Breit-Wheeler process, leading to annihilation of UHE photons, or the pion photoproducton, where single interaction alters the energy of the UHE proton, the single Bethe-Heitler interaction does not change the proton energy significantly. Therefore, unlike all previous processes, the mean free path $\lambda_{B H}$ does not correspond to a horizon. Another quantity is used for this purpose, namely the mean energy loss distance, defined as $\lambda_{B H} \sim[d E /(E c d t)]^{-1}$, where $E$ is the proton energy, which corresponds to the distance on which the energy of the UHE proton is reduced by a factor $e$ due to numerous interactions with background photons $[16,17,20]$. However, it should be emphasized that single Bethe-Heitler interaction deflects the UHE proton by a small angle. This effect is discussed in detail below.

\subsection{Processes involving neutrinos}

UHE neutrinos can be produced either in astrophysical sources, or in some exotic new physics scenarios [21]. Below we compute the horizon due to interaction of UHE neutrinos with cosmic neutrino background $(\mathrm{C} \nu \mathrm{B})$. Following $[22]$ we assume $\mathrm{C} \nu \mathrm{B}$ neutrinos are in their mass states. The cross-section is composed of two parts [22]. The resonant neutrino annihilation occurs in the s-channel:

$$
\nu+\bar{\nu} \longrightarrow Z^{0} \longrightarrow f+\bar{f},
$$

where bar denotes antiparticle, $f$ is a fermion. It has a typical Breit-Wigner shape and is given in the analytic form in [23]. We take the small momentum expansion of the cross-section given by eq. (23) in [23] as

$$
\sigma_{\nu \bar{\nu}}^{R} \simeq 4 \sqrt{2} G_{F} \frac{m_{\nu} M_{Z}^{2} \sqrt{\xi} E}{\left(M_{Z}^{2}-2 E m_{\nu}\right)^{2}+4 E^{2} m_{\nu}^{2} \xi} \mathrm{GeV}^{-2}
$$

where $G_{F}=1.16637 \times 10^{-5} \mathrm{GeV}^{-2}$ is the Fermi's coupling constant, $\xi=\left(\Gamma / M_{Z}\right)^{2}, \Gamma=2.495$ $\mathrm{GeV}$ is the width of $Z^{0}$ resonance and $M_{Z}=91.1876 \mathrm{GeV}$ is the mass of $Z^{0}$ boson, $m_{\nu}$ is neutrino mass, $E$ is energy of UHE neutrino in laboratory frame. Clearly, the position of the resonance scales inversely proportional to the neutrino mass. Throughout this paper we use the reference value $m_{\nu}=0.08 \mathrm{eV} / \mathrm{c}^{2}$, corresponding to the recent cosmological bound from the Planck mission [24], which gives characteristic energy $E_{r}=M_{Z}^{2} c^{2} / 2 m_{\nu} \simeq 5.2 \times 10^{22} \mathrm{eV}$. The amplitude of the resonance does not depend on neutrino mass, and is given by

$$
\sigma_{\nu \bar{\nu}}^{R \max }=2 \sqrt{2} G_{F} M_{Z} / \Gamma \simeq 0.471 \mu b .
$$

The second contribution is the non-resonant cross-section, which is adopted here in the form

$$
\sigma_{\nu \bar{\nu}}^{N R}=\frac{\sigma_{\nu \bar{\nu}}^{h e}}{1+\left(E / E_{r}\right)^{-1}},
$$

where $\sigma_{\nu \bar{\nu}}^{h e} \simeq 8.3 \times 10^{-4} \mu b$.

We assume that neutrino are non-relativistic even at sufficiently high redshift, which is a good approximation for $z<10^{2}$ for $m_{\nu}=0.08 \mathrm{eV} / \mathrm{c}^{2}$. Effects of non-zero momentum on the neutrino annihilation cross-section are studied in [22, 23]. Using the number density of relic neutrinos $n_{C \nu B} \simeq 112 \mathrm{~cm}^{-3}$ and the non-resonant cross-section in the high energy limit one can estimate the horizon for UHE neutrinos at highest energies. Solving the Friedmann equation (see next section) one finds for the redshift $z_{\nu} \simeq 84$. 


\section{The optical depth and the mean free path}

In this section we compute the optical depth for the propagation of UHE particles in the Universe. Imposing the condition that it equals unity we determine the corresponding mean free path. It should be noted that simple estimates, made in the literature, as well as in previous section, do not take into account evolution of $\mathrm{CMB}$ and $\mathrm{C} \nu \mathrm{B}$ fields with time. The simplest way to account for cosmic redshift is to compare this estimate of the mean free path to the expansion scale $c / H_{0}$, where $H_{0}$ is the present day Hubble parameter, see e.g. $[17,20]$. In what follows we describe more rigorous way to take into account both redshift of particle energy as well as the evolution of $\mathrm{CMB}$ and $\mathrm{C} \nu \mathrm{B}$ fields with redshift.

The optical depth along the particle world line $\mathcal{L}$ is defined as

$$
\tau=\int_{\mathcal{L}} \sigma j_{\mu} d x^{\mu}
$$

where $\sigma$ is the cross-section of a given process, $j^{\mu}$ is the 4-current of particles, on which the UHE particle scatters, and $d x^{\mu}$ is the element of the UHE particle world line. We assume the Universe is homogeneous and isotropic, and the background particles are either CMB photons or $\mathrm{C} \nu \mathrm{B}$ neutrinos. Both have thermal distribution functions, given by

$$
f(\mathcal{E} / k T)=\frac{1}{e^{(\mathcal{E}-\mu) / k T} \pm 1},
$$

where $k$ is the Boltzmann constant, $T$ is the $\mathrm{CMB}$ or $\mathrm{C} \nu \mathrm{B}$ temperature, the sign " -" is for photons while the sign "+" is for neutrinos, $\mathcal{E}$ and $\mu$ are the energy and the chemical potential of background particles (for photons $\mu=0$ ). Then the optical depth (3.1) is

$$
\tau(E, t)=\frac{g_{s}}{2 \pi^{2} \hbar^{3} c^{3}} \int_{t}^{0} c d t^{\prime} \int_{\mathcal{E}_{t r}}^{\infty} \mathcal{E}^{2} d \mathcal{E} f(\mathcal{E}) \sigma\left(E, \mathcal{E}, t^{\prime}\right),
$$

where $\mathcal{E}_{t r}$ is threshold energy in a given process, $g_{s}=2$ is the number of helicity states for both protons and neutrinos. Here we assumed that UHE particles move along light-like geodesics. The integral over time can be transformed into the integral over redshift by means of the Friedmann equation. The latter for the flat Universe reads

$$
\left(\frac{1}{a} \frac{d a}{d t}\right)^{2}=\frac{8 \pi G}{3} \rho
$$

where $a$ is the scale factor, $\rho$ is energy density of the Universe, $G$ is Newton's constant. From this equation, the definition of cosmological redshift, as well as the definition of the density parameters

$$
a_{0} / a=1+z, \quad \Omega_{i}=\frac{\rho_{i}}{\rho_{c}}, \quad \rho_{c}=\frac{3 H_{0}^{2}}{8 \pi G},
$$

where $H_{0}$ and $a_{0}$ are present time Hubble parameter and scale factor, respectively, we have

$$
\int_{t}^{0} c d t^{\prime} \longrightarrow \frac{c}{H_{0}} \int_{0}^{z} \frac{d z^{\prime}}{\left(1+z^{\prime}\right) H\left(z^{\prime}\right)},
$$

where $H_{0}$ is the Hubble parameter and the function $H(z)$ is given by

$$
H(z)=\left[\Omega_{r}(1+z)^{4}+\Omega_{M}(1+z)^{3}+\Omega_{\Lambda}\right]^{1 / 2},
$$


and $\Omega_{r}, \Omega_{M}$ and $\Omega_{\Lambda}$ are present densities of radiation, matter and dark energy, respectively. Then the expression (3.3) can be written as follows

$$
\tau(E, z)=\frac{1}{\pi^{2} \hbar^{3} c^{3}} \frac{c}{H_{0}} \int_{0}^{z} \frac{d z^{\prime}}{\left(1+z^{\prime}\right) H\left(z^{\prime}\right)} \int_{\mathcal{E}_{t r}}^{\infty} \mathcal{E}^{2} d \mathcal{E} f(\mathcal{E}) \sigma\left(E, \mathcal{E}, z^{\prime}\right) .
$$

Cosmic expansion results in the energy and temperature dependence on redshift

$$
T=(1+z) T_{0}, \quad \mathcal{E}=(1+z) \mathcal{E}_{0}, \quad E=(1+z) E_{0},
$$

where temperature $T_{0, \gamma} \simeq 2.725 \mathrm{~K}$ for photons, $T_{0, \nu}=(4 / 11)^{1 / 3} \simeq 1.95 \mathrm{~K}$ for neutrinos and energies $E_{0}, \mathcal{E}_{0}$ are measured at the present time.

The second integral in (3.8) can be simplified, provided two conditions are fulfilled: a) the cross-section does not depend on the energy of background particle and b) there is no threshold in the given process $\left(\mathcal{E}_{t r}=0\right)$. In this case one has

$$
\frac{1}{\pi^{2} \hbar^{3} c^{3}} \int_{0}^{\infty} \mathcal{E}^{2} d \mathcal{E} f(\mathcal{E}) \sigma(E, z)=\sigma(E, z) n(z)=\sigma(E, z) n_{0}(1+z)^{3},
$$

where $n_{0}$ is present number density and it stands for either

$$
n_{0, \gamma} \approx \frac{2 \zeta(3)}{\pi^{2}}\left(\frac{\hbar}{m c}\right)^{-3}\left(\frac{k T_{0}}{m_{e} c^{2}}\right)^{3} \simeq 411 \mathrm{~cm}^{-3}
$$

for photons, or $n_{0, \nu}=3 / 4\left(T_{0, \nu} / T_{0, \gamma}\right)^{3} \simeq 113 \mathrm{~cm}^{-3}$ for neutrinos. Then eq. (3.3) becomes

$$
\tau(E, z)=n_{0} \frac{c}{H_{0}} \int_{0}^{z} \frac{\sigma\left(E, z^{\prime}\right)\left(1+z^{\prime}\right)^{2} d z^{\prime}}{H\left(z^{\prime}\right)} .
$$

When the cross-section is just a constant, the integral (3.11) can be readily performed. Assuming $\Omega_{r} \simeq 9.2 \times 10^{-5}, \Omega_{M} \simeq 0.315, \Omega_{\Lambda} \simeq 0.685$ and $H_{0}=67.3 \mathrm{~km} / \mathrm{s} / \mathrm{Mpc}[24]$ in the matter dominated epoch we have

$$
\int_{0}^{z} \frac{\left(1+z^{\prime}\right)^{2} d z^{\prime}}{\left[\Omega_{M}\left(1+z^{\prime}\right)^{3}+\Omega_{\Lambda}\right]^{1 / 2}} \simeq\left\{\begin{array}{cl}
1.045 z, & z \ll 1 \\
1.006 z^{3 / 2}, & z \gg 1 .
\end{array}\right.
$$

The mean free path is defined by the condition $\tau\left(E_{0}, z\right)=1$. For the constant crosssection $\sigma$ at low redshift $z \ll 1$ we get the traditional definition $\lambda=(\sigma n)^{-1}$ used above. For high redshift $z \gg 1$ one can define the redshift, corresponding to the mean free path as

$$
z_{\lambda}=\left(\frac{n_{0} \sigma c}{H_{0}}\right)^{-2 / 3} \simeq 8.9\left(\frac{n_{0}}{n_{0, \gamma}} \frac{\sigma}{10^{-8} \sigma_{T}}\right)^{-2 / 3} .
$$

Using this equation we obtain for UHE neutrinos with highest energies $z_{\lambda} \simeq 84$.

\section{The mean energy loss distance}

When UHE particle annihilates in a given process, such as in the case of Breit-Wheeler one (2.1), the mean free path correpsonds to the horizon defined above.

Another possibility is that the particle is not annihilated in a given process, but scattered, such as in the case of proton producing the pion (2.6). When the energy loss in single 
scattering corresponds to a large fraction of UHE particle energy, the situation is similar to the case of annihilation. However, UHE particle may lose only a small fraction of its energy, as in the case of Bethe-Heitler process (2.8). Here another relevant quantity corresponds the particle horizon defined above is the mean energy loss distance $\tilde{\lambda}$. We define it following [25] as

$$
\tilde{\lambda}^{-1}=\left(\frac{1}{E} \frac{d E}{c d t}\right)
$$

Then we evaluate the quantity

$$
\tilde{\tau}=\int_{t}^{0} \frac{c d t}{\tilde{\lambda}}=\frac{c}{H_{0}} \int_{0}^{z} \frac{d z^{\prime}}{\tilde{\lambda}\left(1+z^{\prime}\right) H\left(z^{\prime}\right)} .
$$

It is computed below for the Bethe-Heitler process.

\section{Results}

Now we apply the method developed in the previous section to the computation of the mean free path for UHE photons, protons and neutrinos, as well as the mean energy loss distance for protons interacting via the Bethe-Heitler process.

\subsection{Photons}

First, we consider cosmic limits on propagation of UHE photons. In the Breit-Wheeler process (2.1) the cross-section depends on both energies through the definition (2.3). When one considers all possible orientations of CMB photons additional averaging over their angular distribution has to be performed [5,6]. The resulting averaged cross section differs from eq. (2.2). The useful approximations for this quanity can be found e.g. in [6, 26, 27]. We use the accurate expression given by eq. (3.23) in Ref. [1]:

$$
\begin{aligned}
\bar{\sigma}_{\gamma \gamma}(x) & =\frac{3}{2} \sigma_{T} \Sigma(x), \quad \Sigma(x)=\frac{1}{x^{2}}\left[\left(x+\frac{1}{2} \log x-\frac{1}{6}+\frac{1}{2 x}\right) \times\right. \\
& \left.\times \log (\sqrt{x}+\sqrt{x-1})-\left(x+\frac{4}{9}-\frac{1}{9 x}\right) \sqrt{1-\frac{1}{x}}\right]
\end{aligned}
$$

We change our variables in eq. (3.8) and get

$$
\tau_{\gamma \gamma}\left(E_{0}, z\right)=\frac{A}{y_{0}^{3}} \int_{0}^{z} \frac{1}{\left(1+z^{\prime}\right)^{4}} \frac{d z^{\prime}}{H\left(z^{\prime}\right)} \int_{1}^{\infty} \frac{x^{2} d x}{\exp (x / y)-1} \Sigma(x),
$$

where

$$
A=\frac{4 \alpha^{2}}{\pi} \frac{c}{H_{0}}\left(\frac{\hbar}{m c}\right)^{-1}\left(\frac{k T_{0}}{m_{e} c^{2}}\right)^{3} \approx 2.37 \times 10^{6}
$$

and

$$
y=y_{0}(1+z)^{2} ; \quad y_{0}=\frac{E_{0}}{m_{e} c^{2}} \frac{k T_{0}}{m_{e} c^{2}},
$$

and $y_{0}$ is the energy $E_{0}$ in units of the critical energy $E_{B W}=\left(m_{e} c^{2}\right)^{2} / k T_{0} \simeq 1.11 \times 10^{15}$ $\mathrm{eV}$. The intergal over energy can be evaluated numerically and we find a reasonable fit

$$
F_{1}(y)=0.839\left(y^{2.1}+2 \times 10^{-8} y^{2.8}\right) \exp \left(-\frac{1.1}{y}\right) .
$$


Then eq. (5.2) becomes

$$
\tau_{\gamma \gamma}\left(y_{0}, z\right)=\frac{A}{y_{0}^{3}} \int_{0}^{z} \frac{1}{\left(1+z^{\prime}\right)^{4}} \frac{d z^{\prime}}{H\left(z^{\prime}\right)} F_{1}\left[y_{0}(1+z)^{2}\right] .
$$

This integral is also evaluated numerically.

In the low energy $E \ll E_{B W}$ and high redshift $z \gg 1$ limit the integral (5.6) can be evaluated analytically. The redshift corresponding to the mean free path in this limit is

$$
z_{\lambda, B W} \simeq 0.21\left(\frac{E}{E_{B W}}\right)^{-1 / 2}
$$

This result is known as the Fazio-Stecker relation [28], see their eq. (9).

In addition to the Breit-Wheeler process (2.1), following [13] we consider also the double pair production process (2.4) with the cross-section defined in (2.5). This process is relevant for the highest energies. The optical depth for this process is

$$
\begin{aligned}
\tau_{\gamma \gamma}^{d p p}\left(y_{0}, z\right) & =\frac{B}{y_{0}^{3}} \int_{0}^{z} \frac{1}{\left(1+z^{\prime}\right)^{4}} \frac{d z^{\prime}}{H\left(z^{\prime}\right)} \int_{2}^{\infty} \sigma_{d p p} \frac{x^{2} d x}{\exp (x / y)-1}= \\
& =\frac{B}{y_{0}^{3}} \int_{0}^{z} \frac{1}{\left(1+z^{\prime}\right)^{4}} \frac{d z^{\prime}}{H\left(z^{\prime}\right)} F_{2}\left[y_{0}\left(1+z^{\prime}\right)^{2}\right]
\end{aligned}
$$

where

$$
\begin{aligned}
F_{2}(y) & =\frac{8}{3}-4 i \pi y-4 y \log \left[\exp \left(\frac{2}{y}\right)-1\right]- \\
& -4 y^{2} \text { PolyLog }\left[2, \exp \left(\frac{2}{y}\right)\right]+2 y^{3} \text { PolyLog }\left[3, \exp \left(\frac{2}{y}\right)\right] .
\end{aligned}
$$

and $B \approx 15.3$.

The condition $\tau\left(y_{0}, z\right)=\tau_{\gamma \gamma}\left(y_{0}, z\right)+\tau_{\gamma \gamma}^{d p p}\left(y_{0}, z\right)=1$ in eqs. (5.2) and (5.8) determines the mean free path of UHE photons. This mean free path is shown in Fig. 1 in megaparsecs and in Fig. 2 in cosmological redshift by the solid curve. The region above the solid curve is opaque for high energy photons. In addition, thick black dashed line shows the boundary of transparency for EBL, according to the baseline model of Inoue et al. [29], while dotted line in Fig. 1 shows the cosmological horizon $z=\infty$.

For the distance smaller than a critical value of about $d_{c}=6.8 \mathrm{kpc}$, the $\mathrm{CMB}$ is transparent to high-energy photons with arbitrary energy. For larger distances there are two branches of solutions for the condition $\tau_{\gamma \gamma}\left(y_{0}, z\right)=1$, respectively corresponding to the different energy-dependence of the average cross-section (5.1). This average cross-section $\bar{\sigma}_{\gamma \gamma}(x)$ increases with the center of mass energy $x$ from the energy threshold $x=1$ to $x \simeq 3.5$, and decreases from $x \simeq 3.5$ to $x \rightarrow \infty$. The energy of the UHE photon corresponding to the critical distance $d_{c}$ is about $1.11 \mathrm{PeV}$, which separates two branches of the solution. The double pair production process (2.4) is relevant for the highest energies, as expected. Photons with energies above $10 \mathrm{PeV}$ are absorbed by the double pair production if they are emitted at redshift above $z \simeq 0.03$ (distance about $120 \mathrm{Mpc}$ ).

For comparison we show by the dashed curve also the mean free path computed at $z=0$, namely neglecting cosmological expansion, see e.g. [13]. 


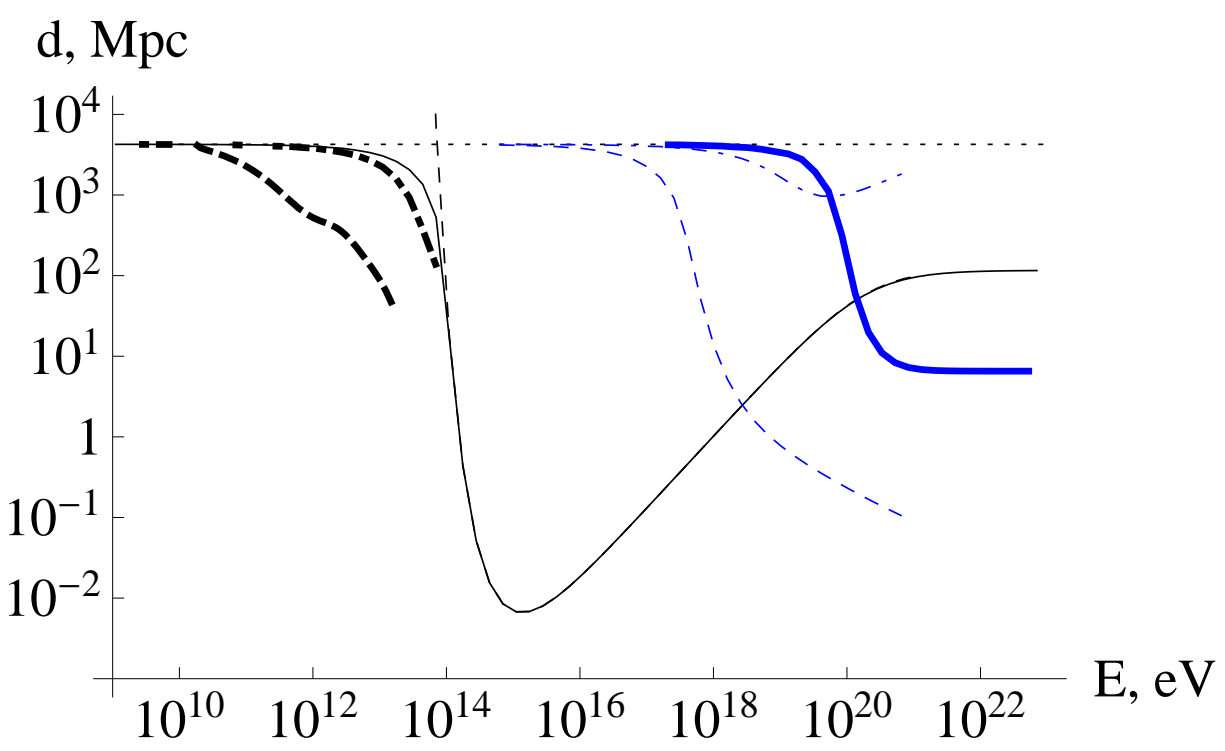

Figure 1. The mean free path, measured in megaparsecs as a function of energy $E$ of UHE particles, measured in electronvolts. In the region above the curves the optical depth is larger than unity. Thin black curve shows the mean free path of UHE photons. Dashed black curve shows the photon mean free path computed without accounting for cosmological evolution (imposing $z=0$ ). Thick blue dashed curve shows the boundary of transparency for extragalactic background light (EBL), according to the baseline model of Inoue et al. [29]. Thick blue curve shows the mean free path of UHE protons (GZK limit). Blue dashed (dotted-dashed) curve shows the mean free path (mean energy loss distance) for UHE protons due to Bethe-Heitler process. Dotted horizontal line shows cosmological horizon.

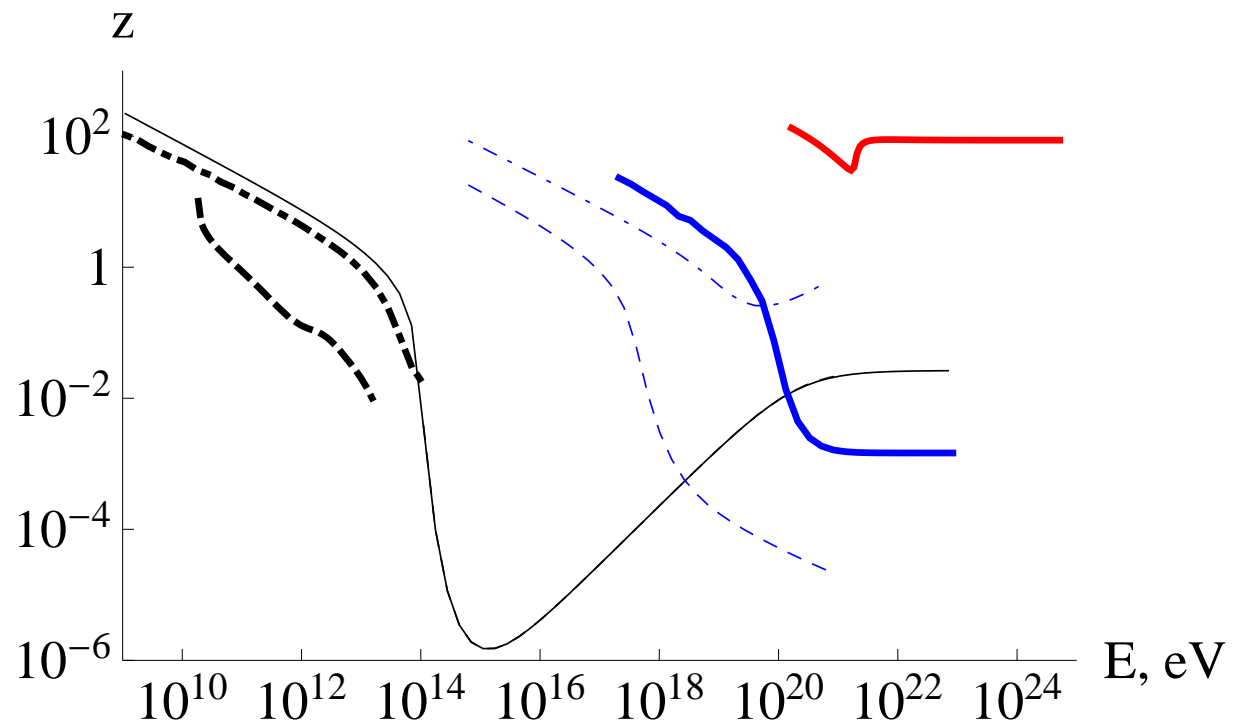

Figure 2. The same as in Fig. 1 for the distance measured in cosmological redshift. Red thick curve shows the mean free path of UHE neutrinos.

We also show by the dashdotted thick curve the mean free path for the photon-photon 
scattering which follows from the Euler-Heisenberg lagrangian, see e.g. [3, 30]. We will discuss this process in a separate publication [31].

Finally, the black dotted thick curve shows the horizon of photons with energies above $20 \mathrm{GeV}$ and below $100 \mathrm{TeV}$, which is determined by their interaction with the EBL. The latest EBL model [29] is used. It is clear, that the contribution of CMB photons gives the absolute upper limit on the mean free path. In the energy range between $1 \mathrm{GeV}$ and $20 \mathrm{GeV}$ the propagation of high energy photons is limited only by the CMB radiation.

\subsection{Protons}

Second, we consider the propagation of UHE protons, accelerated in a source located at a cosmological distance from Earth. First, considering the photopion process (2.6) we use the method developed in the previous section and compute the GZK limit $[14,15]$. This limit applies to protons and other charged particles, leading to the existence of a cutoff in the observed spectrum of (UHE) cosmic rays at about $10^{20} \mathrm{eV}$. For the photopion process (2.6) one can use the simple expression (3.11) with the constant cross-section (2.7). However, we compute the optical depth in the same way as in the case of double pair production, using eq. (5.8) with different value of the constant $B^{\prime} \approx 253$.

The mean free path due to photopion process is shown by the blue thick curve in Fig. 1 in megaparsecs and in Fig. 2 in cosmological redshift. From Fig. 1 it appears that for energies well below $E_{p \gamma} \simeq 3.3 \times 10^{20} \mathrm{eV}$ the GZK limit approaches the cosmological horizon. Instead, from Fig. 2 it follows that the mean free path measured in redshift, below the energy $E_{p \gamma}$, increases with decreasing energy as a power law, which is a consequence of eq. (3.12).

Similarly to the Breit-Wheeler case, the integral (5.8) is evaluated analytically in the low energy $E \ll E_{p \gamma}$ and high redshift $z \gg 1$ limit, with the result

$$
z_{\lambda, G Z K} \simeq 0.57\left(\frac{E}{E_{p \gamma}}\right)^{-1 / 2}
$$

We also evaluate the mean free path due to the Bethe-Heitler process (2.8). Since crosssections (2.9) and (2.10) are given in the proton rest frame, one has to transform photon energy to this reference frame using

$$
\mathcal{E}^{\prime}=2 \Gamma \mathcal{E}=2 \frac{E \mathcal{E}}{m_{p} c^{2}}
$$

where the primed quantity corresponds to the proton rest frame, while unprimed quantities to laboratory reference frame. Then it is convenient to make use of the same type of variable change as before for the Breit-Wheeler process $^{1}$, with a difference that instead of electron mass squared a product of electron and proton masses arises, namely

$$
\bar{x}=2 \frac{E \mathcal{E}}{m_{e} m_{p} c^{4}}, \quad \bar{y}_{0}=2 \frac{E_{0}}{m_{p} c^{2}} \frac{k T_{0}}{m_{e} c^{2}}=\frac{E_{0}}{E_{B H}} .
$$

\footnotetext{
${ }^{1}$ We assume that UHE protons collide with the with CMB photons head on. More accurate calculation with average over angular distribution of the CMB photons does not change qualitatively our results.
} 
The optical depth is computed in the laboratory frame as follows ${ }^{2}$

$$
\begin{aligned}
\tau_{p \gamma}\left(\bar{y}_{0}, z\right) & =\frac{1}{\pi^{2}} \frac{c}{H_{0}}\left(\frac{\hbar}{m c}\right)^{-3}\left(\frac{k T_{0}}{m_{e} c^{2}}\right)^{3} \times \\
& \times \frac{1}{\bar{y}_{0}^{3}} \int_{0}^{z} \frac{1}{\left(1+z^{\prime}\right)^{4}} \frac{d z^{\prime}}{H\left(z^{\prime}\right)} \int_{2}^{\infty} \frac{\bar{x}^{2} d \bar{x}}{\exp (\bar{x} / \bar{y})-1} \sigma_{B H}(x) .
\end{aligned}
$$

The intergal over energy can be evaluated numerically and we find a reasonable fit

$$
F_{3}(\bar{y})=\frac{86.15 \exp \left(-\frac{2}{\bar{y}}\right)}{10^{3 \bar{y}-3.47}+\bar{y}^{-3}} .
$$

Then eq. (5.2) becomes

$$
\tau_{p \gamma}\left(\bar{y}_{0}, z\right)=\frac{C}{\bar{y}_{0}^{3}} \int_{0}^{z} \frac{1}{\left(1+z^{\prime}\right)} \frac{d z^{\prime}}{H\left(z^{\prime}\right)} F_{3}\left[\bar{y}_{0}(1+z)^{2}\right]
$$

where

$$
C=\frac{2 \alpha^{3}}{3 \pi} \frac{c}{H_{0}}\left(\frac{\hbar}{m c}\right)^{-1}\left(\frac{k T_{0}}{m_{e} c^{2}}\right)^{3} \approx 2863 .
$$

The mean free path for protons interacting via the Bethe-Heitler process is shown by blue dashed curve in Fig. 1 in megaparsecs and in Fig. 2 in cosmological redshift. The integral (5.15) is evaluated analytically in the low energy $E \ll E_{B H}$ and high redshift $z \gg 1$ limit, with the result

$$
z_{\lambda, B H} \simeq 0.43\left(\frac{E}{E_{B H}}\right)^{-1 / 2} .
$$

As discussed before, at energies above $10^{18} \mathrm{eV}$ the mean free path is relatively small, about a few hundred kiloparsecs, and it quickly decreases with increasing energy. This is in contrast with the large mean energy loss path, which is above $1 \mathrm{Gpc}$ at energies $10^{18}-10^{20}$ eV, see e.g. $[17,20]$. It means, that before UHE proton starts to lose its energy, it is scattered many hundred times [16]. At each of this scattering the proton recoils, being deflected by a small angle measured in the laboratory reference frame. From the analysis of the cross-section as the function of recoil angle [32], see also [33] in the proton rest frame, it follows that in the high energy limit the photon recoils in the plane, orthogonal to the incident photon. It implies that each scattering produces a deflection of the UHE proton in the laboratory frame by angle $\sim 1 / \gamma$, where $\gamma=E /\left(m_{p} c^{2}\right)$ is proton Lorentz factor. The number of scatterings is given approximately by $\tau$.

One can compute the mean energy loss distance defined in (4.1) and then evaluate the quantity (4.2) using eq. (18)-(20) in [25] to obtain

$$
\tilde{\tau}=\frac{D}{y_{0}^{3}} \int_{0}^{z} \frac{d z^{\prime}}{\left(1+z^{\prime}\right)^{4} H\left(z^{\prime}\right)} \int_{2}^{\infty} \frac{d \bar{x}}{\exp \left(\frac{\bar{x}}{\bar{y}}\right)-1} \phi(\bar{x}),
$$

where the function $\phi(\bar{x})$ is given in eq. (16) in [25]

$$
\phi(\bar{x})=\bar{x}\left[-86.07+50.95 \log \bar{x}-14.45(\log \bar{x})^{2}+2.667(\log \bar{x})^{3}\right]
$$

\footnotetext{
${ }^{2}$ Note that the integral over energy is not transformed to the proton rest frame, as done e.g. in [25]. Instead, only a change of variables is performed in the integral (5.13).
} 


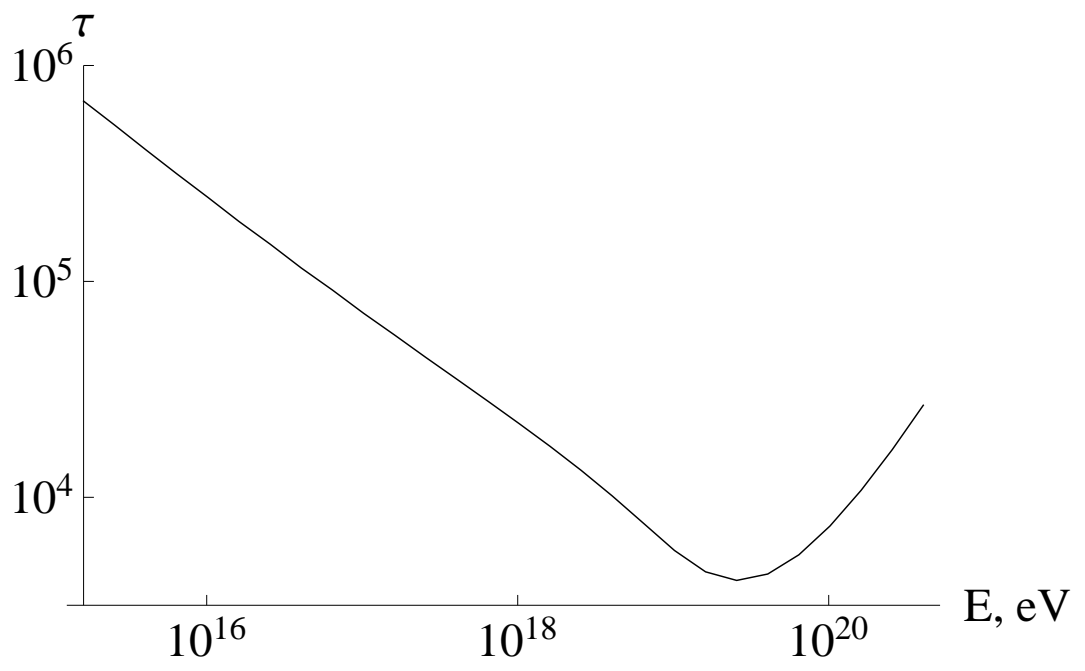

Figure 3. The optical depth at the mean energy distance for the Bethe-Heitler process.

and

$$
D=\frac{2}{\pi^{2}} \alpha^{3} \frac{m_{e}}{m_{p}} \frac{c}{H_{0}}\left(\frac{\hbar}{m c}\right)^{-1}\left(\frac{k T_{0}}{m_{e} c^{2}}\right)^{3} .
$$

From the condition $\tilde{\tau}=1$ we determine the mean energy loss distance $\tilde{\lambda}$. This distance is shown by blue dash-dotted curve in Fig. 1 in megaparsecs and in Fig. 2 in cosmological redshift.

We evaluate the optical depth (5.13) at the redshift, corresponding to $\tilde{\lambda}$ for energies in the range between $10^{15}$ and $10^{20} \mathrm{eV}$ and find it in the range $10^{4}$ to $10^{5}$, see Fig. 3. Since the deflection at each interaction is small, the average number of interactions is proportional to the optical depth. The average deflection angle is then

$$
\delta \sim \frac{\sqrt{\tau(E)}}{\gamma}
$$

We find an average deflection angle of UHE protons as a function of proton energy for sources located at the mean energy loss distance and show it in Fig. 4. At energies $E=10^{16} \mathrm{eV}$, this angle is about $\delta \sim 15^{\prime \prime}$ and it decreases down to $\delta \sim 2.4$ mas for $E=10^{19} \mathrm{eV}$. This latter scale is larger than the angular size of distant blazars [34]. Such deflection, although small compared to deflection in galactic magnetic field [35], inevitably leads to dimming of point sources of UHE protons. It makes also more difficult to detect distant sources.

\subsection{Neutrinos}

Third, we consider the propagation of UHE neutrinos. Such neutrinos can be produced in the source of UHE cosmic rays in decay of secondary pions $\pi^{+} \longrightarrow \mu^{+}+\nu_{\mu}$ or secondary neutrons $n \longrightarrow p+e^{-}+\bar{\nu}_{e}$ [16]. UHE neutrino can be produced also in some extensions of the standard model of particle physics [21]. Such UHE neutrino interacts with the $\mathrm{C} \nu \mathrm{B}$ via the process (2.11). 


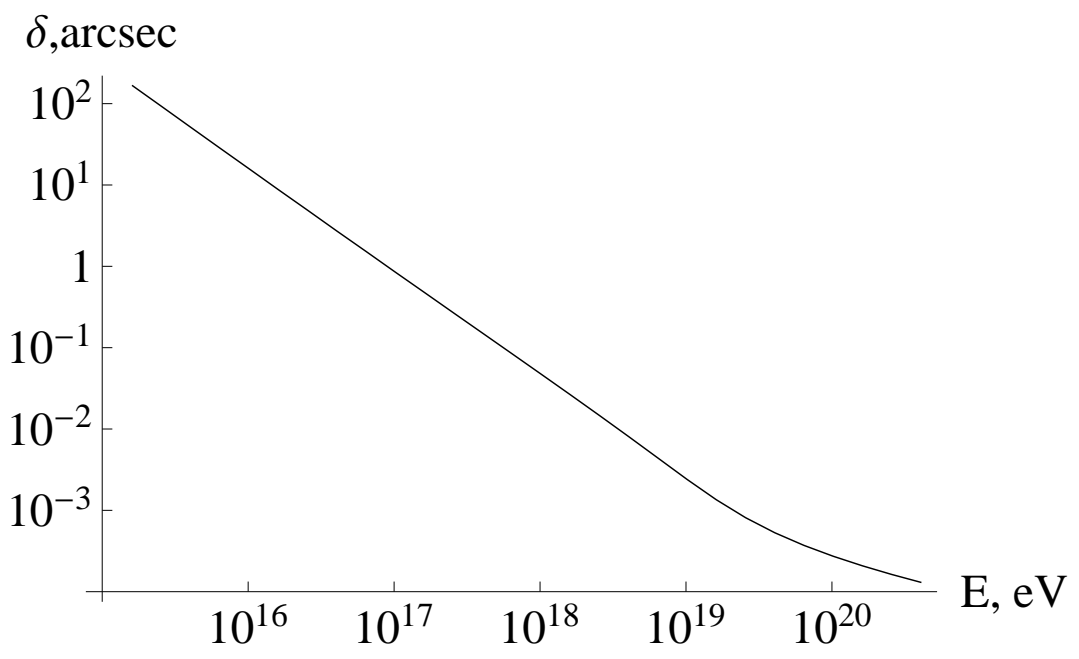

Figure 4. The average deflection angle of UHE protons as function of proton energy for sources located at the mean energy loss distance for the Bethe-Heitler process.

The cross-section of this process has a resonance, and it approaches a constant for highest energies. We compute the optical depth, which instead of eq. (3.8) is given by

$$
\begin{aligned}
\tau_{\nu \nu}(E, z)= & \frac{1}{\pi^{2} \hbar^{3} c^{3}} \frac{c}{H_{0}} \int_{0}^{z} \frac{d z^{\prime}}{\left(1+z^{\prime}\right) H\left(z^{\prime}\right)} \int_{\mathcal{E}_{t r}}^{\infty} \mathcal{E} \sqrt{\mathcal{E}^{2}-\left(m_{\nu} c^{2}\right)^{2}} d \mathcal{E} f(\mathcal{E}) \sigma(E, z) \approx \\
& \frac{1}{\pi^{2} \hbar^{3} c^{3}} \frac{c}{H_{0}} n_{0, \nu} \int_{0}^{z} \frac{\left(1+z^{\prime}\right)^{2} d z^{\prime}}{H\left(z^{\prime}\right)} \sigma(E(1+z \prime)),
\end{aligned}
$$

using the cross-sections given in the laboratory reference frame by eqs. (2.12) and (2.14). The mean free path for neutrinos, measured in cosmological redshift, is shown in Fig. 2 by the thick red curve. Since the characteristic redshifts are high, this curve practically coincides with the horizon, when measured in megaparsecs, so we do not show it in Fig. 1. It is clear that the Breit-Wigner resonance in the cross-section decreases the mean free path in a wide range of energies. The lowest redshift for $E \simeq E_{r}$ at which the Universe is transparent for UHE neutrinos is $z_{\text {min }} \simeq 30$. The resonance produces a dip around $E_{r} /\left(1+z_{\text {min }}\right) \simeq$ $1.7 \times 10^{21}\left(m_{\nu} / 0.08 \mathrm{eV}\right)^{-1} \mathrm{eV}$, where $z_{\min } \simeq 30$. Additional broadening of the resonance, due to thermal effect, is discussed in detail in [22]. At higher energies the corresponding redshift is $z \simeq 87$.

Similarly to the previous cases, in the low energy $E \ll E_{p \gamma}$ and high redshift $z \gg 1$ limit, we find

$$
z_{\lambda, \nu} \simeq 14\left(\frac{E}{E_{r}}\right)^{-2 / 5}
$$

\section{Conclusions}

We reviewed cosmic limits on propagation of ultra high energy particles such as photons, protons and neutrinos, set up by their interactions with the cosmic background of photons and neutrinos. In doing so we take into account explicitly cosmic evolution of both cosmic backgrounds, and redshift of UHE particle energy. This is in contrast with majority of the 
literature, where corresponding mean free paths are found at present epoch, neglecting cosmic expansion. A number of new results were obtained, in particular:

- for UHE photons the contribution of CMB photons gives the absolute upper limit on the mean free path. At high redshift, where other radiation backgrounds, such as EBL are absent, the CMB radiation limits the propagation of UHE photons at energies above $\mathrm{GeV}$.

- for UHE protons the mean free path due to Bethe-Heitler process appears to be much shorter than the mean energy loss distance. This results in multiple deflections suffered by UHE protons, before they start to lose energy in the energy range $10^{16}-10^{20} \mathrm{eV}$. Such deflections result in dimming of point sources of UHE protons, which makes it more difficult to detect them.

- for UHE neutrinos for the first time we compute the horizon as a function of redshift. We found that the Universe is transparent of UHE neutrinos at redshifts $z<30$, near the Breit-Wigner resonance at $E_{r} \simeq 5.2 \times 10^{22}\left(m_{\nu} / 0.08 \mathrm{eV}\right)^{-1} \mathrm{eV}$, and it is transparent at redshifts $z<87$ at higher energies.

- Remarkably, in the low energy and high redshift limit, the Fazio-Stecker relation [28] holds for all processes with exception of neutrinos, and it is given by a universal expression $z_{\lambda} \simeq \mathcal{O}(1)\left(\frac{E}{E_{t h r}}\right)^{-1 / 2}$, where $E_{t h r}$ is the characteristic (e.g. threshold) energy for a given process. In the case of neutrinos similar power law exists $z_{\lambda} \propto E^{-2 / 5}$.

\section{References}

[1] F. A. Aharonian, Very High Energy Cosmic Gamma Radiation. World Scientific, 2003.

[2] M. G. Hauser and E. Dwek, The Cosmic Infrared Background: Measurements and Implications, ARA\&A 39 (2001) 249-307, [astro-ph/0].

[3] R. Ruffini, G. Vereshchagin, and S.-S. Xue, Electron-positron pairs in physics and astrophysics: From heavy nuclei to black holes, Phys. Rep. 487 (Feb., 2010) 1-140, [arXiv:0910.0974].

[4] G. Breit and J. A. Wheeler, Collision of Two Light Quanta, Physical Review 46 (Dec., 1934) 1087-1091.

[5] A. I. Nikishov, Absorption of high-energy photons in the universe, Zhurnal Eksperimental'noi $i$ Teoreticheskoi Fiziki 41 (1961) 549-550.

[6] R. J. Gould and G. P. Schréder, Opacity of the Universe to High-Energy Photons, Physical Review 155 (Mar., 1967) 1408-1411.

[7] V. V. Vassiliev, Extragalactic background light absorption signal in the TeV gamma-ray spectra of blazars., Astroparticle Physics 12 (Jan., 2000) 217-238, [astro-ph/9].

[8] P. S. Coppi and F. A. Aharonian, Understanding the spectra of TeV blazars: implications for the cosmic infrared background, Astroparticle Physics 11 (June, 1999) 35-39, [astro-ph/9].

[9] F. Aharonian, A. G. Akhperjanian, U. Barres de Almeida, A. R. Bazer-Bachi, B. Behera, M. Beilicke, W. Benbow, K. Bernlöhr, C. Boisson, O. Bolz, V. Borrel, I. Braun, E. Brion, A. M. Brown, R. Bühler, T. Bulik, I. Büsching, T. Boutelier, S. Carrigan, P. M. Chadwick, L.-M. Chounet, A. C. Clapson, G. Coignet, R. Cornils, L. Costamante, M. Dalton, B. Degrange, H. J. Dickinson, A. Djannati-Ataï, W. Domainko, L. O'C. Drury, F. Dubois, G. Dubus, J. Dyks, K. Egberts, D. Emmanoulopoulos, P. Espigat, C. Farnier, F. Feinstein, 
A. Fiasson, A. Förster, G. Fontaine, S. Funk, M. Füßling, Y. A. Gallant, B. Giebels, J. F. Glicenstein, B. Glück, P. Goret, C. Hadjichristidis, D. Hauser, M. Hauser, G. Heinzelmann, G. Henri, G. Hermann, J. A. Hinton, A. Hoffmann, W. Hofmann, M. Holleran, S. Hoppe, D. Horns, A. Jacholkowska, O. C. de Jager, I. Jung, K. Katarzyński, E. Kendziorra, M. Kerschhaggl, B. Khélifi, D. Keogh, N. Komin, K. Kosack, G. Lamanna, I. J. Latham, A. Lemière, M. Lemoine-Goumard, J.-P. Lenain, T. Lohse, J. M. Martin, O. Martineau-Huynh, A. Marcowith, C. Masterson, D. Maurin, G. Maurin, T. J. L. McComb, R. Moderski, E. Moulin, M. de Naurois, D. Nedbal, S. J. Nolan, S. Ohm, J.-P. Olive, E. de Oña Wilhelmi, K. J. Orford, J. L. Osborne, M. Ostrowski, M. Panter, G. Pedaletti, G. Pelletier, P.-O. Petrucci, S. Pita, G. Pühlhofer, M. Punch, S. Ranchon, B. C. Raubenheimer, M. Raue, S. M. Rayner, M. Renaud, J. Ripken, L. Rob, L. Rolland, S. Rosier-Lees, G. Rowell, B. Rudak, J. Ruppel, V. Sahakian, A. Santangelo, R. Schlickeiser, F. Schöck, R. Schröder, U. Schwanke, S. Schwarzburg, S. Schwemmer, A. Shalchi, H. Sol, D. Spangler, Ł. Stawarz, R. Steenkamp, C. Stegmann, G. Superina, P. H. Tam, J.-P. Tavernet, R. Terrier, C. van Eldik, G. Vasileiadis, C. Venter, J. P. Vialle, P. Vincent, M. Vivier, H. J. Völk, F. Volpe, S. J. Wagner, M. Ward, A. A. Zdziarski, and A. Zech, New constraints on the mid-IR EBL from the HESS discovery of $V H E$-rays from 1ES 0229+200, A\&A 475 (Nov., 2007) L9-L13, [arXiv:0709.4584].

[10] M. Meyer, M. Raue, D. Mazin, and D. Horns, Limits on the extragalactic background light in the Fermi era, A\&A 542 (June, 2012) A59, [arXiv:1202.2867].

[11] A. Sinha, S. Sahayanathan, R. Misra, S. Godambe, and B. S. Acharya, Estimation of the Extragalactic Background Light Using TeV Observations of BL Lac Objects, ApJ 795 (Nov., 2014) 91, [arXiv:1409.3693].

[12] R. W. Brown, K. O. Mikaelian, and R. J. Gould, Absorption of High-Energy Cosmic Photons through Double-Pair Production in Photon-Photon Collisions, Astrophys. Lett. 14 (1973) 203.

[13] P. S. Coppi and F. A. Aharonian, Constraints on the Very High Energy Emissivity of the Universe from the Diffuse GeV Gamma-Ray Background, ApJ 487 (Sept., 1997) L9+, [astro-ph/9].

[14] K. Greisen, End to the Cosmic-Ray Spectrum?, Physical Review Letters 16 (Apr., 1966) $748-750$.

[15] G. T. Zatsepin and V. A. Kuz'min, Upper Limit of the Spectrum of Cosmic Rays, Soviet Journal of Experimental and Theoretical Physics Letters 4 (Aug., 1966) 78.

[16] C. D. Dermer and A. Atoyan, Ultra-high energy cosmic rays, cascade gamma rays, and high-energy neutrinos from gamma-ray bursts, New Journal of Physics 8 (July, 2006) 122, [astro-ph/0606629].

[17] V. S. Berezinskii and S. I. Grigor'eva, A bump in the ultra-high energy cosmic ray spectrum, A\&A 199 (June, 1988) 1-12.

[18] H. Bethe and W. Heitler, On the Stopping of Fast Particles and on the Creation of Positive Electrons, Royal Society of London Proceedings Series A 146 (Aug., 1934) 83-112.

[19] M. J. Chodorowski, A. A. Zdziarski, and M. Sikora, Reaction rate and energy-loss rate for photopair production by relativistic nuclei, ApJ 400 (Nov., 1992) 181-185.

[20] T. Stanev, R. Engel, A. Mücke, R. J. Protheroe, and J. P. Rachen, Propagation of ultrahigh energy protons in the nearby universe, Phys. Rev. D 62 (Nov., 2000) 093005, [astro-ph/0003484].

[21] A. Ringwald, Super-GZK neutrinos, Journal of Physics Conference Series 39 (May, 2006) 393-399.

[22] C. Lunardini, E. Sabancilar, and L. Yang, Ultra high energy neutrinos: absorption, thermal effects and signatures, Journal of Cosmology and Astroparticle Physicsjcap 8 (Aug., 2013) 14, [arXiv: 1306.1808]. 
[23] J. C. D'Olivo, L. Nellen, S. Sahu, and V. van Elewyck, UHE neutrino damping in a thermal gas of relic neutrinos, Astroparticle Physics 25 (Feb., 2006) 47-56, [astro-ph/0507333].

[24] Planck Collaboration, P. A. R. Ade, N. Aghanim, C. Armitage-Caplan, M. Arnaud, M. Ashdown, F. Atrio-Barandela, J. Aumont, C. Baccigalupi, A. J. Banday, and et al., Planck 2013 results. XVI. Cosmological parameters, A\&A 571 (Nov., 2014) A16, [arXiv:1303.5076].

[25] G. R. Blumenthal, Energy Loss of High-Energy Cosmic Rays in Pair-Producing Collisions with Ambient Photons, Phys. Rev. D 1 (Mar., 1970) 1596-1602.

[26] F. A. Aharonian, A. M. Atoian, and A. M. Nagapetian, Photoproduction of electron-positron pairs in compact X-ray sources, Astrofizika 19 (Apr., 1983) 323-334.

[27] P. S. Coppi and R. D. Blandford, Reaction rates and energy distributions for elementary processes in relativistic pair plasmas, MNRAS 245 (Aug., 1990) 453-507.

[28] G. G. Fazio and F. W. Stecker, Predicted High Energy Break in the Isotropic Gamma Ray Spectrum: a Test of Cosmological Origin, Nature 226 (Apr., 1970) 135-+.

[29] Y. Inoue, S. Inoue, M. A. R. Kobayashi, R. Makiya, Y. Niino, and T. Totani, Extragalactic Background Light from Hierarchical Galaxy Formation: Gamma-Ray Attenuation up to the Epoch of Cosmic Reionization and the First Stars, ApJ 768 (May, 2013) 197, [arXiv: 1212.1683].

[30] V. B. Berestetskii, E. M. Lifshitz, and V. B. Pitaevskii, Quantum Electrodynamics. Elsevier, 1982.

[31] S. Batebi, S. Tizchang, R. Ruffini, G. Vereshchagin, and S.-S. Xue, Euler-heisenberg interaction and opacity of the universe at high energy, in preparation (2015).

[32] R. Jost, J. M. Luttinger, and M. Slotnick, Distribution of Recoil Nucleus in Pair Production by Photons, Physical Review 80 (Oct., 1950) 189-196.

[33] J. W. Motz, H. A. Olsen, and H. W. Koch, Pair Production by Photons, Reviews of Modern Physics 41 (Oct., 1969) 581-639.

[34] M. Giroletti, Millimeter-VLBI observations of blazars, Journal of Physics Conference Series 131 (Oct., 2008) 012054.

[35] G. A. Medina Tanco, E. M. de Gouveia Dal Pino, and J. E. Horvath, Deflection of Ultra-High-Energy Cosmic Rays by the Galactic Magnetic Field: From the Sources to the Detector, ApJ 492 (Jan., 1998) 200-204, [astro-ph/9707041]. 